\title{
A New Strategy of Validities' Computation for Multimodel Approach: Experimental Validation
}

\author{
Abdennacer BEN MESSAOUD \\ National Engineering School of \\ Tunis, LR11ES20 LACS Laboratory \\ Tunis El Manar University, Tunisia
}

\author{
Samia TALMOUDI BEN AOUN \\ National Engineering School of \\ Tunis, LR11ES20 LACS Laboratory \\ Tunis El Manar University, Tunisia
}

\author{
Moufida LAHMARI KSOURI \\ National Engineering School of \\ Tunis, LR11ES20 LACS Laboratory \\ Tunis El Manar University, Tunisia
}

\begin{abstract}
The evaluation of validities is a fundamental step in the design of the multimodel approach. Indeed, it is thanks to validities that we estimate the contribution of each base-model in the reproduction of the behavior of the global process in a given operating area. These coefficients are calculated most commonly by the approach of the residues formulated by the distance between the real output and the sub-models' outputs. In this paper, a strategy allowing to improve the performances of the residues' approach in terms of precision and robustness is proposed. This strategy is based on a quasi-hierarchical structuring. A simulation example and a validation on a semibatch reactor showed the interest and the effectiveness of the proposed strategy.
\end{abstract}

Keywords-Validities; residues' approach; multimodel; quasihierarchical structuring; experimental validation

\section{INTRODUCTION}

The multimodel approach has been of considerable interest for many years. The works of [1] and [2] define the idea of the multimodel approach as the apprehension of a nonlinear behavior of a system by a set of local models (linear or affine) characterizing the system operation in different operating zones. The motivation of this approach ensues from the fact that it is often difficult to conceive or to identify a model taking into account the complexity of the studied system. Using this definition, the multimodels can be understood as models defined around different operating points.

The multimodel approach offers an interesting alternative and a powerful tool to bypass the difficulties to identify, control and diagnose a nonlinear and complex system [3]-[8].

The multimodel modeling concept consists of simply to represent the dynamics of a nonlinear system by a family of relatively simple models properly characterizing the functioning of the system in its different operating areas. This models family constitutes the base of local models of the system.

In the literature, three different methods may be employed for the determination of the models' base [9]. The first one is only based on the measures of inputs/outputs of the system from which are estimated the different models' parameters [5],
[10], [11]. For the second and the third method, we supposed to have a nonlinear mathematical model, the base-models are obtained either by linearization around the different operating points [1], or by convex polytopic transformation [6], [12].

The global model output, so-called multimodel output, is obtained by a combination of the local models' outputs weighted by their validity indexes representing the relevance degree of each model estimated at each instant by a suitable decision process.

Several validities' calculation methods have been proposed in the literature [13]-[17]. The residues' approach is the most commonly used [3], [4], [12], [13], [18]-[20]. This approach is based on the calculation of the residues formulated by the distance between the real output and the sub-models' outputs. However, the performances of the multimodel approach, whose base-models' validities are calculated by the residues' approach, are considerably deteriorated in several cases of complex systems [13], [16], [17]. A new strategy, presented in this paper, allows to solve this problem and to improve the precision of the multimodel approach.

The paper is organized as follow: In Section 2, the validity concept is presented. The classical residues' approach is presented in Section 3. The new strategy of validities' computation by the residues' approach is detailed in Section 4 . To illustrate the interest and efficiency of the new strategy, the simulation results are given in Section 5. Section 6 is reserved for validation on a semi-batch reactor.

\section{VALIDITY CONCEPT}

The validity $v_{i}$ represents the contribution of the local model $M_{i}$ in the description of the behavior of the global system in a given operating area. It is estimated at each instant by a suitable decision process (Fig. 1). When it is equal to 0 , the corresponding model is considered as inactive and consequently has no influence on the global multimodel system. If, on the contrary, this validity takes the value 1 , the model represents perfectly the process at the considered instant. In the case where the relevance degree is between 0 and 1 , the corresponding model $M_{i}$ represents partially the system behavior [13]. 


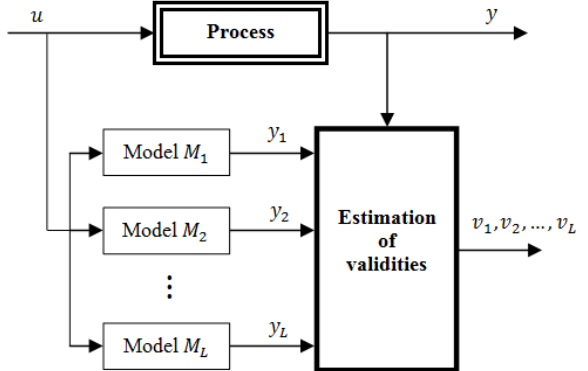

Fig. 1. Structure of the validities' estimation for the multimodel approach.

The base-models' validities satisfy the following convex sum property [21]:

$$
\left\{\begin{array}{l}
v_{i} \in[0,1] ; i=1,2, \ldots, L \\
\sum_{i=1}^{L} v_{i}=1
\end{array}\right.
$$

Where, $v_{i}$ is the validity of the $i^{\text {th }}$ model and $L$ is the number of base-models.

Once the validities are estimated, the multimodel output is obtained by a combination of the local models' outputs weighted by their respective validities and given by the following formula:

$$
y_{m m}(k)=\sum_{i=1}^{L} v_{i}(k) y_{i}(k)
$$

Where, $y_{i}$ is the output of local model $M_{i}$.

\section{RESIDUES' APPROACH}

This method requires only knowledge of the base-models outputs and the global system response [14].

The validities' calculation is based on the residues which are based on the online calculation of the difference between the process output and those of the various models $M_{i}$ of the base:

$$
r_{i}=\left|y-y_{i}\right| ; i=1, \ldots, L
$$

Where, $y$ is the process output and $y_{i}$ is the output of the model $M_{i}$.

The validities are deducted from the following equation:

$$
v_{i}=1-\frac{r_{i}}{\sum_{j=1}^{L} r_{i}} ; i=1, \ldots, L
$$

These validities are normalized by (5).

$$
v_{i}^{\text {simp }}=\frac{v_{i}}{\sum_{j=1}^{L} v_{i}} ; i=1, \ldots, L
$$

The validities' approach presented above is effective in cases where the operating areas present overlapping [10].

In some cases the validities values are so moved closer that we need to implement methods known as reinforcement methods in order to distinguish them. This reinforcement operation may be defined for example by (6).

$$
v_{i}^{\text {renf }}=v_{i} \prod_{\substack{j=1 \\ j \neq i}}^{L}\left(1-v_{j}\right) ; i=1, \ldots, L
$$

The normalized reinforced validities, satisfying the convex sum property, are given by [3]:

$$
v_{i}^{\text {renf }}{ }_{n}=\frac{v_{i}^{\text {renf }}}{\sum_{j=1}^{L} v_{j}^{\text {renf }}} ; i=1, \ldots, L
$$

\section{THE NEW PROPOSED STRATEGY}

\section{A. Problem Statement}

In order to highlight the deterioration phenomenon of the quality of the approximation by the multimodel representation using the residues' approach to estimate the validities' indexes, the approximation problem of the following static nonlinear function is considered:

$$
y(t)=1+\exp \left(-t^{2}\right) \sin (\pi t) ; t \in[-2 ; 2]
$$

The system operating space can be decomposed into two operating areas. Each zone is then characterized by a submodel (Fig. 2):

$$
\left\{\begin{array}{l}
\text { Model } M_{1}: y_{1}(t)=3.03 t+1 \\
\text { Model } M_{2}: y_{2}(t)=-1.78 t+2.74
\end{array}\right.
$$

The multimodel is then given by the following equation:

$$
y_{m m}(t)=v_{1}(t) y_{1}(t)+v_{2}(t) y_{2}(t)
$$

whose validities are estimated by the residues' approach.

The Fig. 3 illustrates a dynamic behavior of the nonlinear function badly approached by the multimodel in the range $[0.1,0.7]$. This is due to the insufficiency of the decomposition of the operating space into two zones. Decomposing then the operating space into three zones (Fig. 4), a third model is added to the models' base:

$$
\text { Model } M_{3}: y_{3}(t)=-1.82 t-0.77
$$




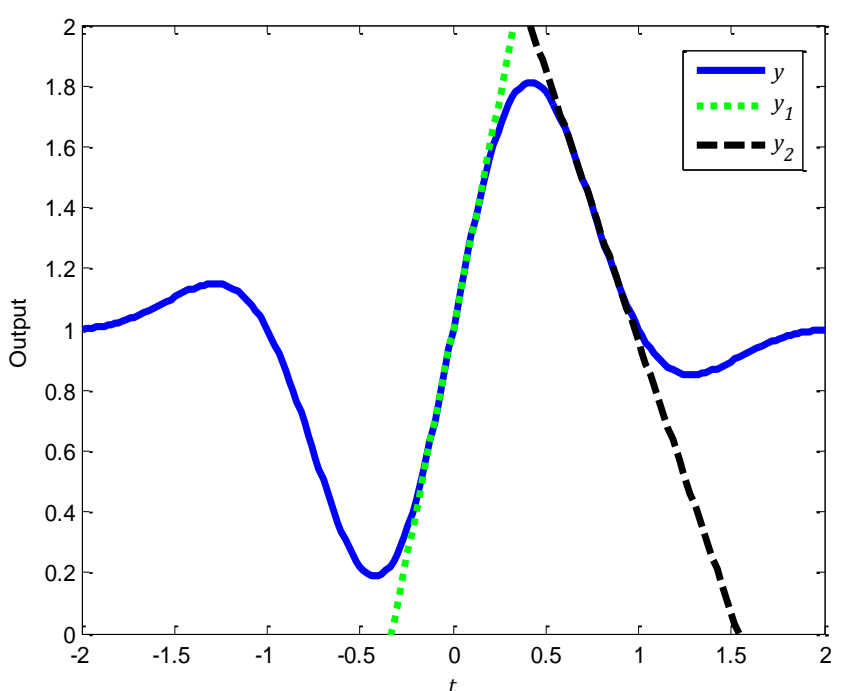

Fig. 2. Nonlinear system and local models with $L=2$.

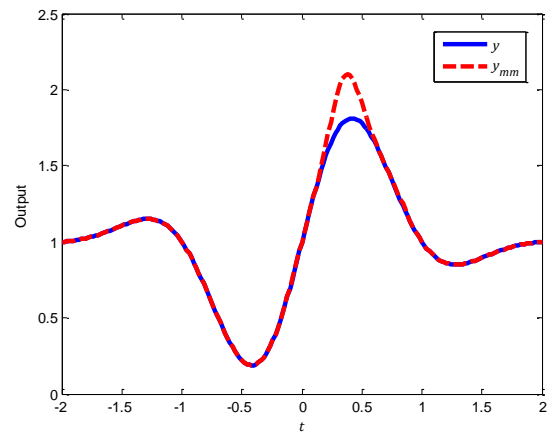

Fig. 3. Nonlinear system and multimodel approximation with $L=2$.

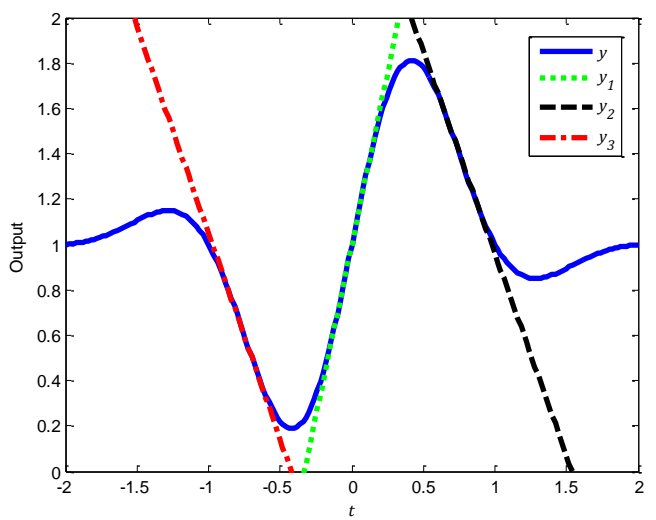

Fig. 4. Nonlinear system and local models with $\mathrm{L}=3$.

Re-calculating the relevance degrees of the different submodels by the residues' approach, the result of approximation by the multimodel approach is given in Fig. 5. This figure shows a high deterioration of the approximation quality compared to the case when the operating space is decomposed into two areas.

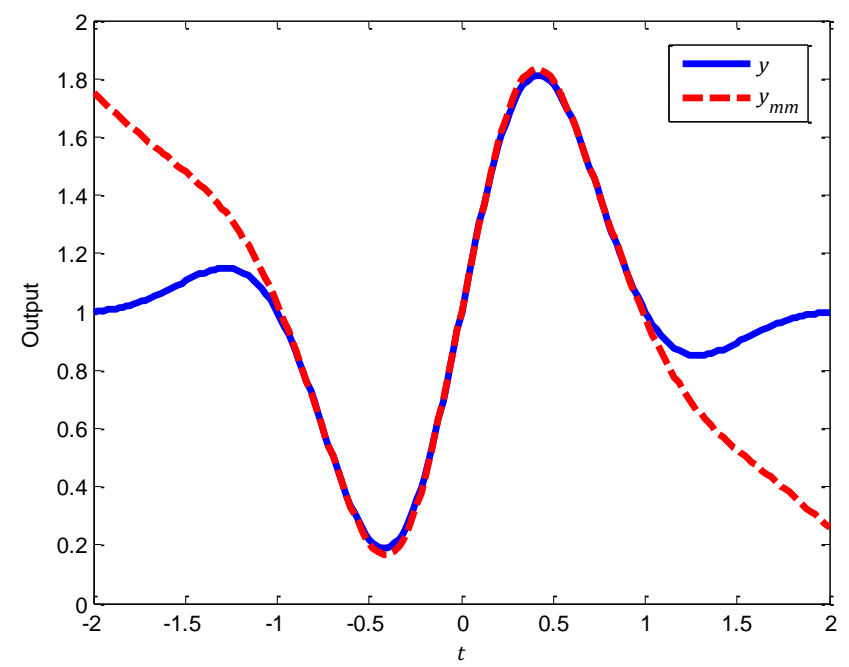

Fig. 5. Nonlinear system and multimodel approximation with $\mathrm{L}=3$.

By examining the strategy on which is based the estimation of the validities values by residues' approach, it can be concluded that the weakness of this strategy is related to the normalization phase relative to the different residues. Indeed, this phase does not take into account the result obtained in the case of the decomposition of the operating space into two areas. To remedy this problem a new strategy will be proposed in the following.

\section{B. New Proposed Strategy}

Let us assume that at instant $k$, the residues' calculation using (3) gives ascending values $\left(r_{1} \leq r_{2} \leq \cdots \leq r_{L}\right)$. The new strategy is based on a quasi-hierarchical structuring as shown in Fig. 6 whose validities $\left(v_{1}^{\prime}, v_{2}^{\prime}, \ldots, v_{L}^{\prime}\right)$ and $\left(v_{\text {pmm }}, v_{\text {pmm }_{2}}, \ldots, v_{\text {pmm }_{(L-2)}}\right)$ are calculated by (5) or (7).

The validity of each base-model is given, therefore, by the following equation:

$$
\left\{\begin{array}{l}
v_{1}=v_{1}^{\prime} \prod_{j=1}^{L-2} v_{p m m_{j}} \\
v_{i}=v_{i}^{\prime} \prod_{j=i-1}^{L-2} v_{p m m_{j}} ; i=2, \ldots,(L-1) \\
v_{L}=v_{L}^{\prime}
\end{array}\right.
$$




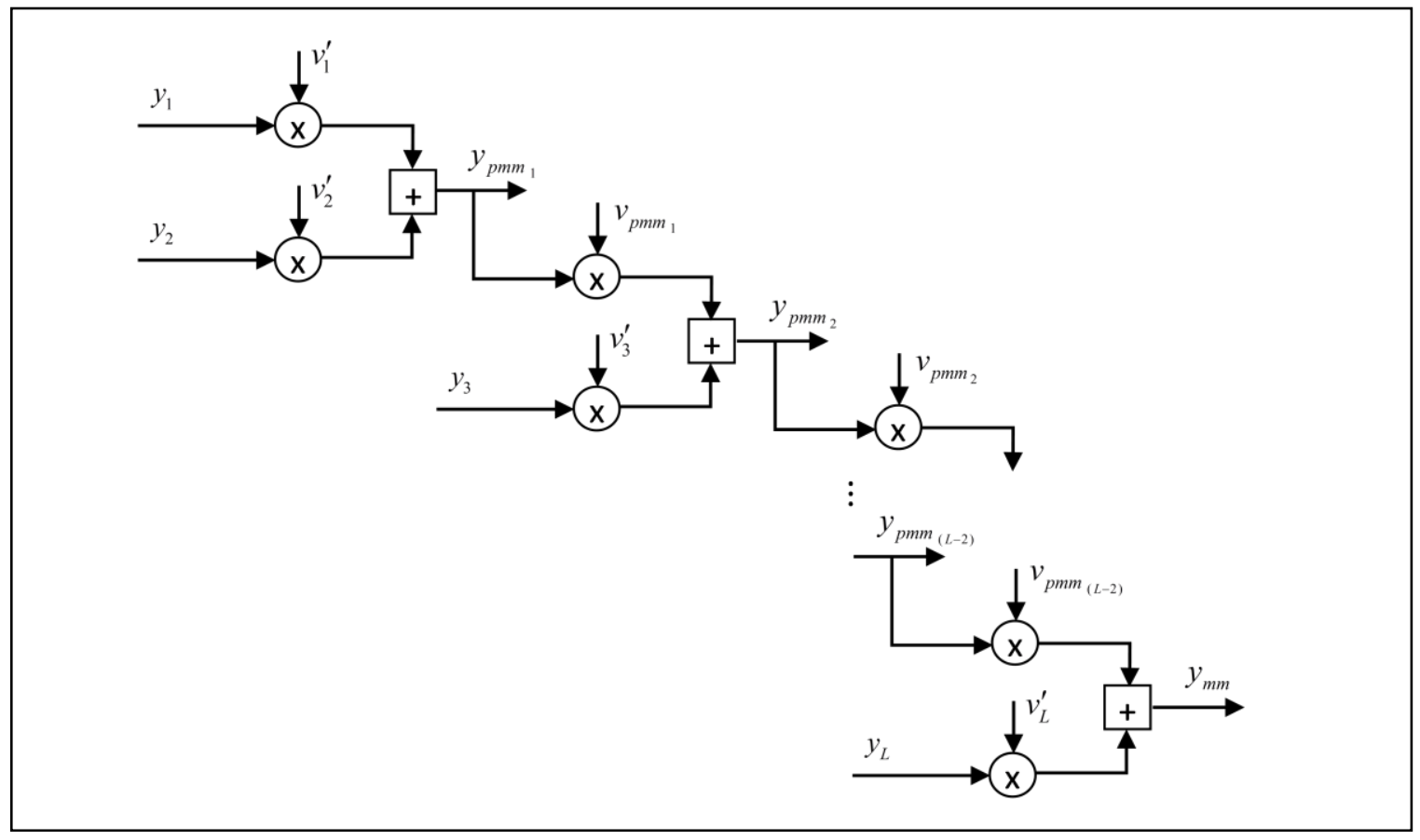

Fig. 6. New strategy: quasi-hierarchical structure.

In the general case and for each instant $k$, the base-models validities are calculated by Algorithm 1.

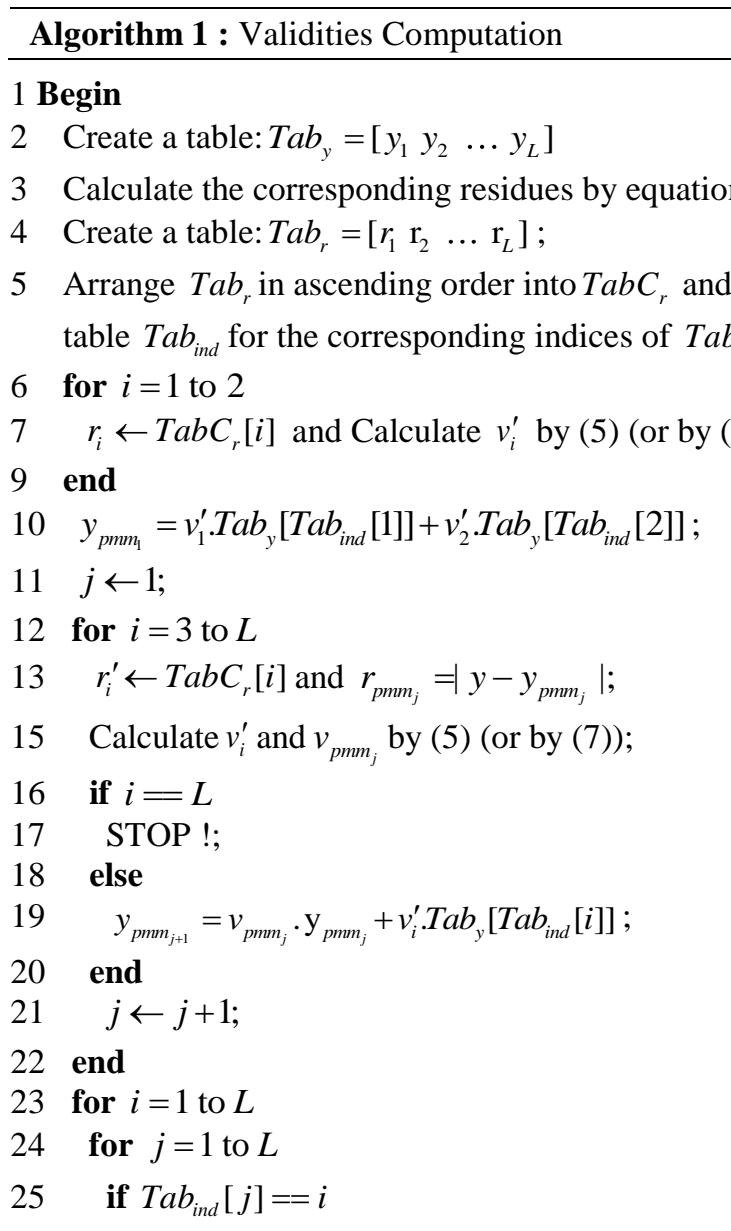

$$
\begin{aligned}
& 26 \quad \text { if } j==1 \\
& 27 \quad v_{i}=v_{j}^{\prime} \prod_{k=1}^{L-2} v_{p m m_{k}} ; \\
& 28 \quad \text { else if } j=L \\
& \quad v_{i}=v_{L}^{\prime} ; \\
& 29 \quad \text { else } \\
& 30 \quad v_{i}=v_{j}^{\prime} \prod_{k=j-1}^{L-2} v_{p m m_{k}} ; \\
& 31 \quad \text { end } \\
& 32 \quad \text { end } \\
& 33 \quad \text { end } \\
& 34 \text { end } \\
& 35 \text { end }
\end{aligned}
$$

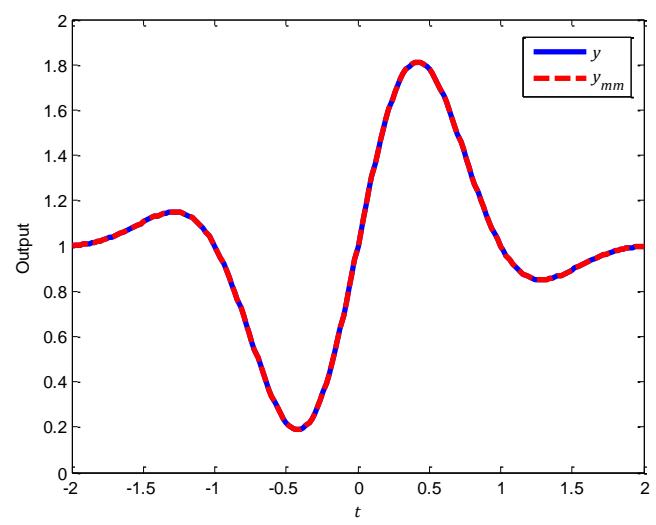

Fig. 7. Nonlinear system and multimodel approximation with $L=3$ (new strategy). 
Applying this new strategy to the static nonlinear function (8), a perfect adequacy between the nonlinear model data and those of the multimodel is shown in Fig. 7.

\section{SimUlATION EXAMPLE}

In order to underline the interest of the new strategy of residues' approach for validities' computation, a simulation example was considered. The performances of the models are assessed using the Mean Square Error (MSE) and the Variance-Accounted-For (VAF) indicators given by the following equations [22]:

$$
\begin{gathered}
M S E=\frac{1}{N} \sum_{k=1}^{N}\left(y(k)-y_{m m}(k)\right)^{2} \\
V A F=\max \left\{1-\frac{\operatorname{var}\left(y(k)-y_{m m}(k)\right)}{\operatorname{var}(y(k))}, 0\right\} \times 100 \%
\end{gathered}
$$

Where, $y(k)$ and $y_{m m}(k)$ are the system and the multimodel output, and $\operatorname{var}(\cdot)$ denotes the variance of a signal.

The considered example is a discrete system with time varying parameters, described by the following equation [17]:

$$
\begin{array}{r}
y(k)=-a_{1}(k) y(k-1)-a_{2}(k) y(k-2)+ \\
+b_{1}(k) u(k-1)+b_{2}(k) u(k-2)
\end{array}
$$

The variation laws of different parameters of the process are given by Fig. 8 .

By applying the multimodel approach, Talmoudi et al. [17] demonstrated that the models base is composed of three models whose transfer functions are given by:

$$
\begin{aligned}
& H_{1}\left(z^{-1}\right)=\frac{0.18104 z^{-1}+0.07183 z^{-2}}{1-1.1657 z^{-1}+0.2073 z^{-2}} \\
& H_{2}\left(z^{-1}\right)=\frac{0.10423 z^{-1}+0.1325 z^{-2}}{1-1.2806 z^{-1}+0.3258 z^{-2}} \\
& H_{3}\left(z^{-1}\right)=\frac{0.018301 z^{-1}+0.20512 z^{-2}}{1-1.3801 z^{-1}+0.42936 z^{-2}}
\end{aligned}
$$
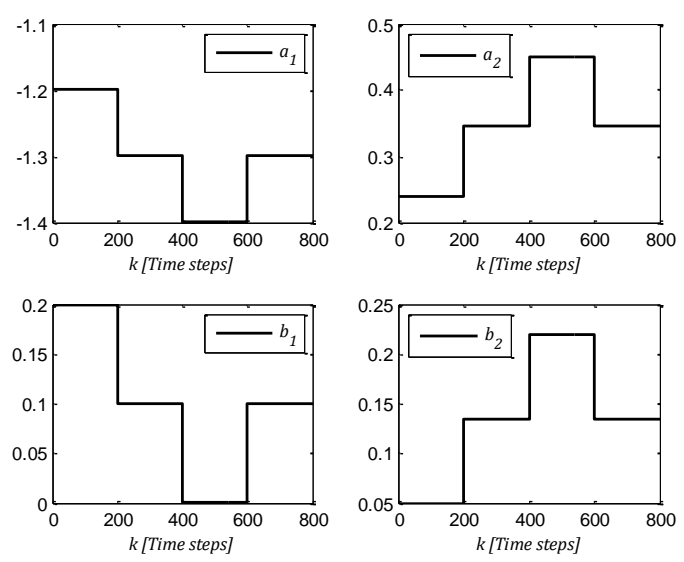

Fig. 8. Variation laws of different process parameters.
The multimodel output is obtained by the fusion of the different models outputs $y_{1}(k), y_{2}(k)$ and $y_{3}(k)$ weighted by their respective validity indexes $v_{1}(k), v_{2}(k)$ and $v_{3}(k)$ :

$$
y_{m m}(k)=v_{1}(k) y_{1}(k)+v_{2}(k) y_{2}(k)+v_{3}(k) y_{3}(k)
$$

Let us consider the following validation input sequence:

$$
u(k)=0.5+(\exp (-0.05 k)) \cos \left(\frac{k \pi}{30}\right)
$$

The validities are calculated at first by the classical formulation of the residues' approach (reinforced validities), and secondly by the new strategy.

The simulation results are given in Fig. 9 and 10 where the relative error between the real and the multimodel outputs is calculated using the following equation:

$$
e_{\text {relative }}(k)=\left|\frac{y(k)-y_{m m}(k)}{y(k)}\right| \times 100 \%
$$

These figures show that the new strategy of residues' approach for validities' computation clearly improves the precision of the multimodel output compared with the results obtained by the residues' approach in its classical formulation. This is also proved by the performance indicators (Table 1).

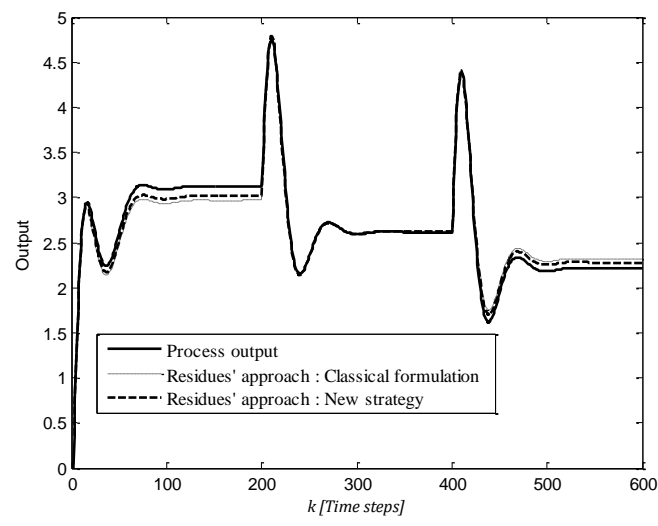

Fig. 9. Real and multimodel outputs.

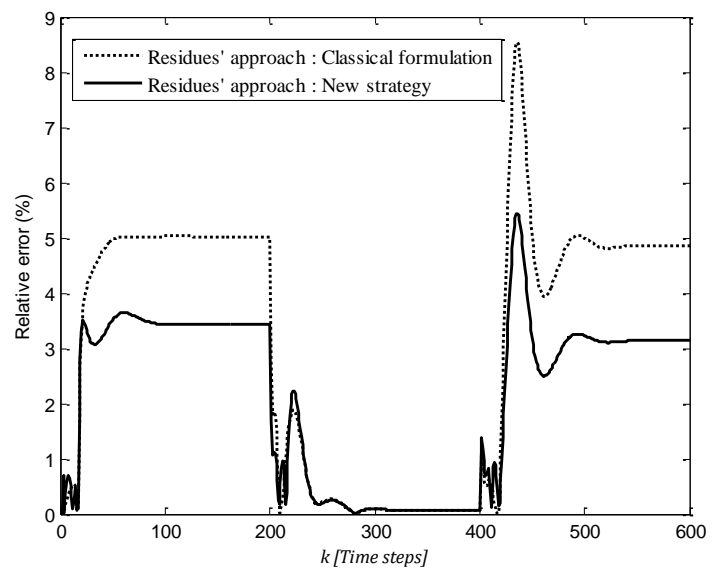

Fig. 10. Evolutions of the relative errors. 
TABLE I. MSE AND VAF

\begin{tabular}{|l|l|l|}
\hline & Classical formulation & New strategy \\
\hline $\boldsymbol{M S E}$ & 0.0104 & 0.0049 \\
\hline $\boldsymbol{V A F}$ & $97.07 \%$ & $98.65 \%$ \\
\hline
\end{tabular}

\section{A. Robustness Study}

In order to evaluate the quality of the validities calculation method based on the new strategy while comparing it to the residues' approach in its classical formulation, a robustness study of these two methods was made. Three cases are examined:

1) Robustness relative to output measurement error:

In this case, the variance of the measurement error was adjusted so that a noise-to-signal ratio at output (SNR) was $0.1 \%, 1 \%, 5 \%$ and $10 \%$ in power:

$$
\operatorname{SNR}(\%)=\frac{\operatorname{var}(w(k))}{\operatorname{var}\left(y_{0}(k)\right)} \times 100
$$

Where, $y_{0}(k)$ represents the part of the noise-free output signal and $w(k)$ is the measurement error.

2) Robustness relative to the base-models parameters:

The deviation of the base-models parameters is defined as follows:

$$
\left\{\begin{array}{l}
a_{i}=a_{i_{0}}+\Delta_{a_{i}} \\
b_{i}=b_{i_{0}}+\Delta_{b_{i}}
\end{array}\right.
$$

with:

$$
\left\{\begin{array}{l}
\Delta_{a_{i}}= \pm \frac{\delta a_{i}}{100}\left|a_{i_{0}}\right| \\
\Delta_{b_{i}}= \pm \frac{\delta b_{i}}{100}\left|b_{i_{0}}\right|
\end{array}\right.
$$

The simulation was made for the following variations:

$$
\left\{\begin{array}{l}
\delta a_{i}=0.01 ; 0.1 ; 0.2 \text { and } 0.5 \% \\
\delta b_{i}=0.01 ; 0.1 ; 0.2 \text { and } 0.5 \%
\end{array}\right.
$$

3) Robustness relative to the base-models poles:

The deviation of the base-models poles is defined as follows:

$$
p_{i}=p_{i_{0}}+\Delta_{p_{i}}
$$

with:

$$
\Delta_{p_{i}}= \pm \frac{\delta p_{i}}{100}\left|p_{i_{0}}\right|
$$

The simulation was made for the following variations: $\delta p_{i}=0.01 ; 0.1 ; 0.2$ and $0.5 \%$

By examining Tables 2 to 4, it can be noted that despite the influence of the validities calculation method based on the new strategy by the level of the measurement error as well as the deviations of the parameters and poles of the base-models, it generally gives better results than the classical residues method.

TABLE II. ROBUSTNESS RELATIVE TO OUTPUT MEASUREMENT ERROR

\begin{tabular}{|l|l|l|l|}
\hline & SNR $(\%)$ & Classical formulation & New strategy \\
\hline \multirow{4}{*}{$\boldsymbol{M S E}$} & 0.1 & 0.0109 & 0.0054 \\
\cline { 2 - 4 } & 1 & 0.0170 & 0.0105 \\
\cline { 2 - 4 } & 5 & 0.0320 & 0.0244 \\
\cline { 2 - 4 } & 10 & 0.0441 & 0.0372 \\
\hline \multirow{4}{*}{$\boldsymbol{V A F}$} & 0.1 & $96.93 \%$ & $98.49 \%$ \\
\cline { 2 - 4 } & 1 & $95.25 \%$ & $97.07 \%$ \\
\cline { 2 - 4 } & 5 & $91.32 \%$ & $93.38 \%$ \\
\cline { 2 - 4 } & 10 & $87.69 \%$ & $89.62 \%$ \\
\hline
\end{tabular}

TABLE III. RoBustNess RELATIVE TO THE BASE-MOdels PARAMETERS

\begin{tabular}{|l|l|l|l|}
\hline \multirow{4}{*}{$\boldsymbol{M} \boldsymbol{N} \boldsymbol{E}$} & $\begin{array}{l}\text { Deviation of } \\
\text { parameters } \\
(\%)\end{array}$ & $\begin{array}{l}\text { Classical } \\
\text { formulation }\end{array}$ & New strategy \\
\cline { 2 - 4 } & 0.01 & 0.0105 & 0.0048 \\
\cline { 2 - 4 } & 0.1 & 0.0328 & 0.0239 \\
\cline { 2 - 4 } & 0.2 & 0.0511 & 0.0406 \\
\hline \multirow{4}{*}{ VAF } & 0.5 & 0.0227 & 0.0183 \\
\cline { 2 - 4 } & 0.01 & $96.99 \%$ & $98.61 \%$ \\
\cline { 2 - 4 } & 0.1 & $90.62 \%$ & $93.26 \%$ \\
\cline { 2 - 4 } & 0.2 & $90.30 \%$ & $92.56 \%$ \\
\hline
\end{tabular}

TABLE IV. ROBUSTNESS RELATIVE TO THE BASE-MODELS POLES

\begin{tabular}{|l|l|l|l|}
\hline & $\begin{array}{l}\text { Deviation of } \\
\text { the poles (\%) }\end{array}$ & $\begin{array}{l}\text { Classical } \\
\text { formulation }\end{array}$ & New strategy \\
\hline \multirow{4}{*}{ MSE } & 0.01 & 0.0099 & 0.0046 \\
\cline { 2 - 4 } & 0.1 & 0.0030 & 0.0014 \\
\cline { 2 - 4 } & 0.2 & 0.0285 & 0.0212 \\
\cline { 2 - 4 } & 0.5 & 0.0660 & 0.0600 \\
\hline \multirow{4}{*}{ VAF } & 0.01 & $97.18 \%$ & $98.72 \%$ \\
\cline { 2 - 4 } & 0.1 & $99.18 \%$ & $99.63 \%$ \\
\cline { 2 - 4 } & 0.2 & $94.52 \%$ & $96.29 \%$ \\
\cline { 2 - 4 } & 0.5 & $81.14 \%$ & $82.72 \%$ \\
\hline
\end{tabular}

\section{VALIDATION ON A CHEMICAL REACTOR}

The performances obtained by the new proposed validities calculation strategy incited us to apply it on a real model of chemical reactor [5], [11]. 
Fig. 11 shows the experimental device of the process.

The used reactor is a semi-batch reactor for the chemical esterification of the olive oil according to the following reaction:

\section{Acid + Alcohol $\rightleftarrows$ Ester + Water}

The esterification reaction is carried out in a stirred tank surrounded by a jacket where a heat-transfer fluid which assures a thermal contribution to the reactor flows at a constant rate.

The heat-transfer fluid passes through a plate heat exchanger (E2) where it will be cooled, then through a resistor exchanger (E1) where it will be heated before arriving at the jacket.

Temperature sensors are used to measure the temperatures of the reactor $\left(\mathrm{T}_{\mathrm{r}}\right)$ and those of the heat transfer fluid at the inlet $\left(\mathrm{T}_{\text {ede }}\right)$ and outlet $\left(\mathrm{T}_{\text {sde }}\right)$ of the jacket.

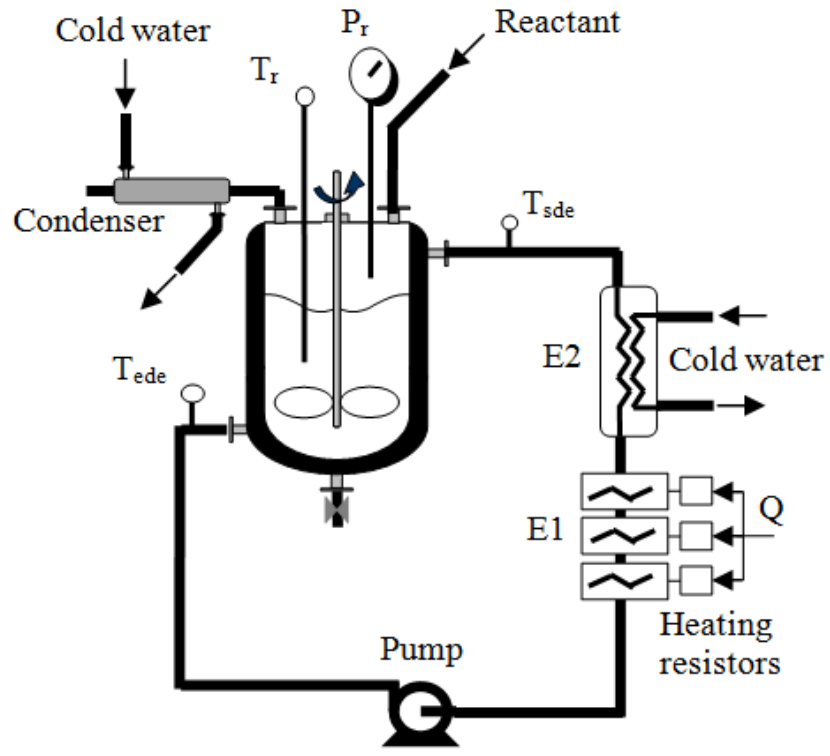

Fig. 11. Experimental device.

Three operational phases are distinguished in the production of ester:

- Heating phase: The reactive initially at an ambient temperature is heated to a temperature which corresponds to the reaction temperature.

- Reaction phase: During which the temperature of the reactional environment is maintained constant.

- Cooling phase: In order to retrieve the ester, the reactor is cooled back to the ambient temperature.

The process is considered as a mono-variable system where the control variable is the electric power Q supplied by the heating resistors while the output is the reactor temperature $\mathrm{T}_{\mathrm{r}}[5]$.

Such a system is nonlinear and the use of multimodel approach is recommended [11].
Fig. 12 represents the set of identification data picked out of the reactor. The selected excitation signal Q is a Pseudo Random Binary Sequence (PRBS) applied to the reactor with a sampling time equal to $180 \mathrm{~s}$.

The models' base is determined by applying the method based on the Kohonen networks [11]. This approach requires firstly determining the number of clusters. The next step consists in classifying the identification data set. And finally a step of structural and parametric estimation of the base-models is necessary.

Three second order systems are obtained:

$$
\begin{aligned}
& H_{1}\left(z^{-1}\right)=\frac{5.95 \times 10^{-5} z^{-1}+0.00185 z^{-2}}{1-1.238 z^{-1}+0.2646 z^{-2}} \\
& H_{2}\left(z^{-1}\right)=\frac{-3.255 \times 10^{-5} z^{-1}+0.00113 z^{-2}}{1-0.7956 z^{-1}-0.1906 z^{-2}} \\
& H_{3}\left(z^{-1}\right)=\frac{9.959 \times 10^{-5} z^{-1}+0.0018 z^{-2}}{1-1.135 z^{-1}+0.1677 z^{-2}}
\end{aligned}
$$
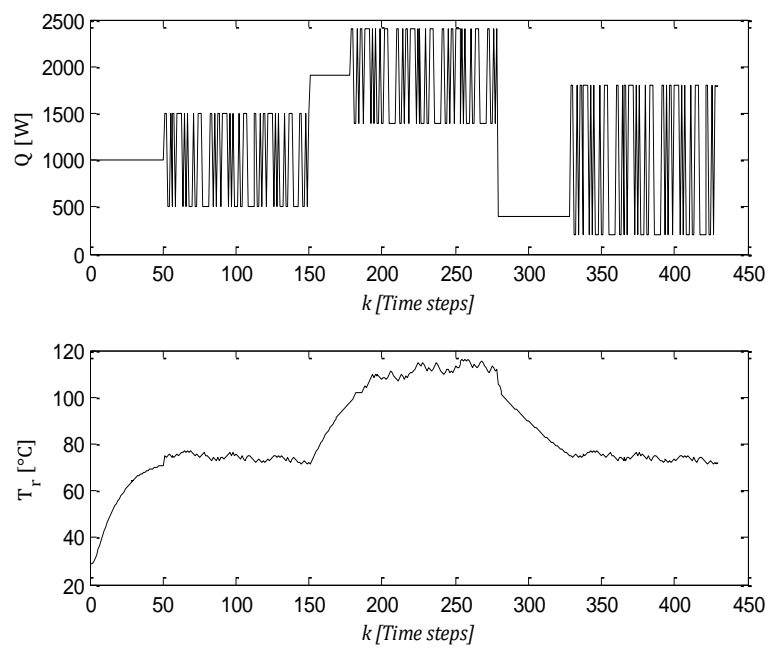

Fig. 12. Identification data set.

The result of the validation phase is given by Fig. 13 where the multimodel output is calculated by the fusion of the three base-models outputs weighted by their respective validity indexes determined at first by the residues' approach in its classical formulation (simple validities), and secondly by the new proposed strategy.

It can be seen that the new strategy of validities' computation by residues' approach offers a very satisfactory precision as compared to the residues' approach in its classical formulation. Indeed, the multimodel output, obtained by exploiting this new strategy, follows with a high precision the real output and describes perfectly the system behavior. However, the output obtained by the exploitation of the classical residues' approach follows the real output with a relatively important error. This is also proved on Fig. 14. On this figure, we drew the evolutions of the relative errors between the system output and the multimodel outputs 
exploiting the residues' approach in its classical formulation and the new proposed strategy. It's clearly observed that the relative error is equal to zero by applying the new proposed strategy.

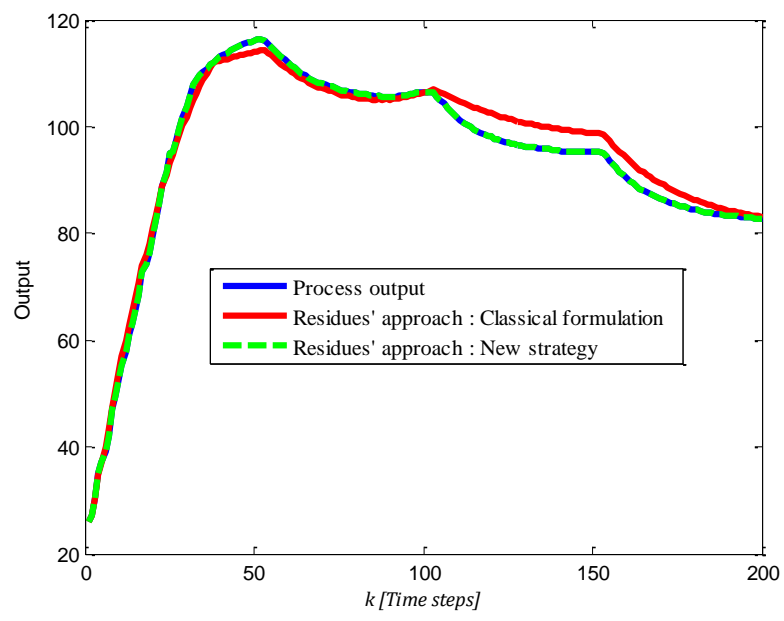

Fig. 13. Real and multimodel outputs of chemical reactor.

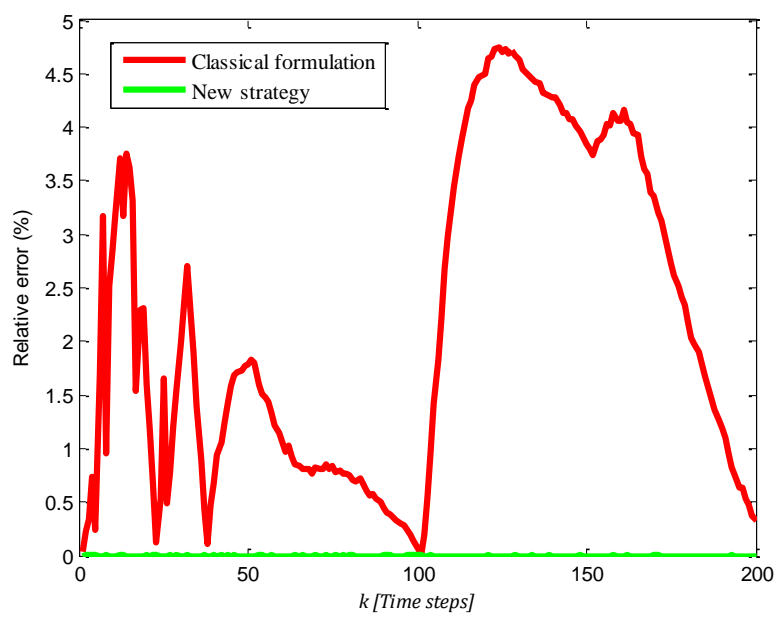

Fig. 14. Evolutions of the relative errors of chemical reactor.

TABLE V. MSE AND VAF OF CHEMICAL REACTOR

\begin{tabular}{|l|l|l|}
\hline & Classical formulation & New strategy \\
\hline $\boldsymbol{M S E}$ & 6.1632 & $8.116 \times 10^{-29}$ \\
\hline $\boldsymbol{V A F}$ & $98.48 \%$ & $100 \%$ \\
\hline
\end{tabular}

The performance indicator MSE (Mean Square Error) was calculated to evaluate the new proposed strategy of validities' computation by residues' approach compared to the classical formulation of the same approach. It is null in the case of applying the new proposed strategy (Table 5). The varianceaccounted-for $V A F$ asserts this result by its value that is equal to $100 \%$ (Table 5).

\section{CONCLUSION}

This paper treats one of the principal issues of the multimodel approach which is the base-models validities estimation. In this study, a new strategy, allowing to improve the performances of the residues' approach for validities' computation, is proposed. This strategy is based on a quasihierarchical structuring. The different steps of validities computation were detailed. The numerical simulation results, described in this paper, prove the efficiency of the new proposed strategy as well as its impact on the improvement of the performances of the residues' approach in terms of precision and robustness. The use of the new strategy on a model of semi-batch chemical reactor showed that, in this case, the multimodel approach leads to a perfect modeling of the real process.

Operating modes management and resolving conflicting connections between them are meaningful and challenging issues, which will be studied in the future work.

\section{REFERENCES}

[1] T. A. Johansen and B. A. Foss, "Nonlinear Local Model Rrepresentation for Adaptative System", IEEE International Conference on intelligent control and instrumentation, Singapore, vol. 2, 1992, pp.677-682.

[2] R. Murray-smith and T. A. Johansen, Multiple Model Appraoches to Modelling and control, Taylor \& Francis, London, 1997.

[3] N. Elfelly, J. Y. Dieulot, M. Benrejeb, and P. Borne, "Multimodel Control Design using Unsupervised Classifiers", Studies in Informatics and Control, vol. 21, no. 1, 2012, pp. 101-108.

[4] A. Karoui and M. Ksouri, "Diagnosis by the Multimodel Approach in the Frequency Domain", 4th International Conference on Logistics (LOGISTIQUA) Proccedings, Hammamet, Tunisia, 2011, pp. 261-267.

[5] M. Ltaief, A. Messaoud, and R. Ben Abdennour, "Optimal Systematic Determination of Models' Base for Multimodel Representation: Real Time Application", International Journal of Automation and Computing, vol. 11 , no. 6 , 2014, pp. 644-652.

[6] A. M. Nagy, G. Mourot, B. Marx, J. Ragot, and G. Schutz, "Systematic Multimodeling Methodology Applied to an Activated Sludge Reactor Model", American Chemical Society: Industrial \& Engineering Chemistry Research ,vol. 49, no. 6, 2010, pp. 2790-2799.

[7] C. Othman, I. Ben Cheikh, and D. Soudani, "On the Internal MultiModel Control of Uncertain Discrete-Time Systems", International Journal of Advanced Computer Science and Applications (ijacsa), vol. 7, no. 9, 2016. http://dx.doi.org/10.14569/IJACSA.2016.070912.

[8] H. Bouzaouache, "Output Feedback Controller Synthesis for DiscreteTime Nonlinear Systems", International Journal of Advanced Computer Science and Applications (ijacsa), vol. 8, no. 4, 2017. http://dx.doi.org/10.14569/IJACSA.2017.080441

[9] M. Chadli and P. Borne, Multiple Models Approach in Automation: Takagi-Sugeno Fuzzy Systems, Wiley-ISTE, 2013.

[10] N. Elfelly, J. Y. Dieulot, and P. Borne, "A Neural Approach of Multimodel Representation of Complex Processes", International Journal of Computers, Communications and Control, vol. 3, no. 2, 2008, pp. 149-160.

[11] S. Talmoudi, K. Abderrahim, R. Ben Abdennour, and M. Ksouri, "Multimodel Approach using Neural Networks for Complex Systems Modeling and Identification”, Nonlinear Dynamics and Systems Theory, vol. 8, no. 3, 2008, pp. 299-316.

[12] A. Salem, A. S. Tlili, and N. B. Braiek, "On the Plotytopic and Multimodel State Observers of Inductor Motor", Journal of Automation \& Systems Engineering, vol. 2, no. 4, 2008.

[13] R. Ben mohamed, H. Ben Nasr, and F. M'sahli, "A Multimodel Approach for a Nonlinear System based on Neural Network Validity", International Journal of Intelligent Computing and Cybernetics, vol. 4, no. 3, 2011, pp. 331-352.

[14] F. Delmotte, Analyse Multi-modèle, PhD Thesis, Université des sciences et techniques de Lille, France, 1997.

[15] M. Ksouri, Contributions à la Commande Multimodèle des Processus Complexes, PhD Thesis, Université des sciences et techniques de Lille, France, 1999. 
[16] M. Ltaief, K. Abderrahim, R. Ben Abdennour, and M. Ksouri, “A Fuzzy Fusion Strategy for the Multimodel Approach Application", WSEAS Transactions on Circuits and Systems, vol. 2, no. 4, 2003, pp.686-691.

[17] S. Talmoudi, K. Abderrahim, R. Ben Abdennour, and M. Ksouri, "A New Technique of Validities' Computation for Models' Base", WSEAS Transactions on Circuits and Systems, vol. 2, no. 4, 2003, pp. 680-685.

[18] S. Bedoui, M. Ltaief, and K. Abderrahim, "New Method for the Systematic Determination of the Model's Base of Time Varying Delay System", International Journal of Computer Applications, vol. 46, no. 1, 2012, pp. 13-19.

[19] I. Chihi, A. Abdelkrim, and M. Benrejeb, "Multi-model Approach to Characterize Human Handwriting Motion", Biol Cybern, vol. 110, no. 1, 2016, pp. 17-30.
[20] A. Sassi and A. Abdelkrim, "New Stability Conditions for Nonlinear Systems described by Multiple Model Approach”, International Journal of Electrical and Computer Engineering (IJECE), vol. 6, no. 1, 2016, pp. 177-187.

[21] R. Orjuela, B. Marx, J. Ragot, and D. Maquin, "State Estimation of Nonlinear Systems based on Heterogeneous Multiple Models: some Recent Theoretical Results", 7th Workshop on Advanced Control and Diagnosis, Zielona Gora, Poland, 2009.

[22] R. Orjuela, B. Marx, J. Ragot, and D. Maquin, "Nonlinear System Identification using Heterogeneous Multiple Models", International Journal of Applied Mathematics and Computer Science, vol. 23, no. 1, 2013, pp. 103-115. 